Cahiers $d u$ MONDE RUSSE

\section{Cahiers du monde russe}

Russie - Empire russe - Union soviétique et États indépendants

45/3-4 | 2004

Varia

\title{
Quelques traits spécifiques du symbolisme russe
}

\section{Catherine Depretto}

\section{OpenEdition \\ Journals}

Édition électronique

URL : https://journals.openedition.org/monderusse/8704

DOI : 10.4000/monderusse. 8704

ISSN : $1777-5388$

\section{Éditeur}

Éditions de l'EHESS

\section{Édition imprimée}

Date de publication : 1 juillet 2004

Pagination : $579-590$

ISBN : 2-7132-2009-2

ISSN : $1252-6576$

\section{Référence électronique}

Catherine Depretto, "Quelques traits spécifiques du symbolisme russe », Cahiers du monde russe [En ligne], 45/3-4 | 2004, mis en ligne le 01 janvier 2007, consulté le 04 septembre 2022. URL : http:// journals.openedition.org/monderusse/8704; DOI : https://doi.org/10.4000/monderusse.8704 
chercher : repérer : avancer

Cet article est disponible en ligne à l'adresse :

http://www.cairn.info/article.php?ID REVUE=CMR\&ID NUMPUBLIE=CMR 453\&ID ARTICLE=CMR 4530579

\title{
Quelques traits spécifiques du symbolisme russe
}

\author{
par CATHERINE DEPRETTO
}

\section{Editions de l'EHESS | Cahiers du monde russe}

\author{
2004/3-4 - Vol 45 \\ ISSN 1252-6576 | ISBN 2713220092 | pages 579 à 590
}

Pour citer cet article :

—DEPRETTO C., Quelques traits spécifiques du symbolisme russe, Cahiers du monde russe 2004/ 3-4, Vol 45, p. 579590.

Distribution électronique Cairn pour les Editions de l'EHESS.

(C) Editions de l'EHESS. Tous droits réservés pour tous pays.

La reproduction ou représentation de cet article, notamment par photocopie, n'est autorisée que dans les limites des conditions générales d'utilisation du site ou, le cas échéant, des conditions générales de la licence souscrite par votre établissement. Toute autre reproduction ou représentation, en tout ou partie, sous quelque forme et de quelque manière que ce soit, est interdite sauf accord préalable et écrit de l'éditeur, en dehors des cas prévus par la législation en vigueur en France. Il est précisé que son stockage dans une base de données est également interdit. 


\section{QUELQUES TRAITS SPÉCIFIQUES DU SYMBOLISME RUSSE}

Dans l'histoire de la poésie française, le symbolisme se laisse difficilement définir. $\mathrm{Au}$ sens strict, il désigne un courant poétique mineur qui n'a duré que quelques années et n'a pas à alléguer de grand nom. Lancé en 1886 par le manifeste de Jean Moréas (1856-1910), le mouvement faisait du symbole le signe de ralliement d'une réaction idéaliste contre le naturalisme et le Parnasse. Mais le terme, utilisé de manière extensive, tend aussi à désigner la modernité poétique française, prise entre romantisme et surréalisme, et finit par englober toute une lignée de poètes, allant de Baudelaire à Valéry. En définitive, le symbolisme ne désignerait pas quelque chose de très précis pour la poésie française et l'on aurait un peu de mal à s'entendre sur son identité : «mot vague pour un mouvement introuvable. »1

En Russie, le symbolisme renvoie à un phénomène important qui a marqué la vie littéraire russe plus de vingt ans et reste la principale école poétique du $\mathrm{XX}^{\mathrm{e}}$ siècle. Même si d'autres courants, dont les principaux sont l'acméisme et le futurisme, émergent dans les années 1910, il est facile de montrer leur filiation avec le symbolisme, malgré les déclarations tonitruantes de leurs représentants. Aussi préfère-ton englober aujourd'hui ces mouvements dans la catégorie générale du postsymbolisme et désigner l'ensemble de la période par le terme de « modernisme ». De toute façon, en termes de rayonnement et d'importance, tous ces -ismes ne sont pas comparables : mouvement complexe et contradictoire, qui connaît différentes phases de développement et rassemble au moins deux générations principales d'écrivains, le symbolisme s'impose en terrain russe comme le grand courant ; son caractère décisif pour l'histoire de la poésie russe ne fait aucun doute ; la validité du terme n'est pas remise en question.

En Russie - et c'est la première différence essentielle avec la situation française -, le symbolisme est avant tout synonyme d'essor, de renouveau poétique. Après l'épanouissement du début du XIX'e siècle, centré autour de la figure

1. Bertrand Marchal, Lire le Symbolisme, Paris, Dunod, 1993, p. XI. 
de Puškin, la poésie russe était tombée en demi-sommeil. Lorsque, vers les années 1890, de nouvelles aspirations commencent à émerger, celles-ci se tournent vers les pratiques poétiques françaises dans leur recherche de modèles ${ }^{2}$. La période du symbolisme naissant a ceci de commun avec les autres périodes de mutation culturelle en Russie qu'elle reçoit une impulsion décisive de l'étranger et s'appuie sur une pratique systématique de la traduction. Dans cette volonté de sortir du cadre spécifiquement russe, de renouer avec l'ouverture européenne de la littérature russe du début du siècle ${ }^{3}$, réside un premier trait essentiel du symbolisme, qui marquera toute son histoire, même si, à partir des années 1910, font surface des orientations ouvertement néo-slavophiles et nationalistes. Ainsi le symbolisme russe n'est-il pas tant un mouvement, une nouvelle école que l'annonce d'une véritable mutation culturelle; quelque chose de très important commence à bouger, à changer dans la conception même de la littérature, disons pour simplifier qu'est en train de se constituer pour la première fois en Russie un véritable champ littéraire, distinct du politique et du social.

D'emblée, on remarque aussi une différence capitale avec la France, différence qui renvoie au décalage général de la littérature russe par rapport à l'Europe. Au sens strict, le symbolisme en France est une réponse au Parnasse, à la rigidité de ses formes ; en Russie, les deux sont réunis, et dans les aspirations des premiers symbolistes, chez Brjusov en particulier, beaucoup d'éléments renvoient au Parnasse. Ainsi, les successeurs des symbolistes reprendront-ils à leur compte chacune de ces composantes, pour l'acméisme - le Parnasse et pour le futurisme, paradoxalement, - la composante symboliste proprement dite (rôle de la sensation pure, phonolâtrie de Kručenyh, etc.).

Mutation culturelle plus que simple école, le symbolisme russe est à ses débuts volontairement et systématiquement orienté vers la culture européenne contemporaine et, en premier lieu, vers la poésie française, de Baudelaire à Mallarmé. Si Mallarmé et Rimbaud restent relativement dans l'ombre ${ }^{4}$, Baudelaire est beaucoup traduit en Russie et très tôt, d'abord par des gens qui n'ont rien de symboliste et l'interprètent comme un critique de la société bourgeoise, puis par les symbolistes, aussi bien ceux de la première que de la seconde génération, Bal'mont, Brjusov, Annenskij mais aussi Ivanov, et surtout Ellis ${ }^{5}$. Pour la première génération, sont particulièrement significatifs les thèmes baudelairiens du rejet du quotidien, de la recherche des paradis artificiels, de l'individualisme élitiste, de l'érotisme, du mal... Pour la seconde génération, le sonnet «Correspondances » prend une valeur emblématique : il est une sorte de quintessence du baudelairisme et, pour les

2. Voir l'ouvrage fondateur de Georgette Donchin, The influence of French symbolism on Russian poetry, La Haye, Mouton, 1958.

3. Ibid.

4. Exception faite, pour Rimbaud, du sonnet « Voyelles ». Pour Mallarmé, voir R. Doubrovkine, Stéphane Mallarmé i Rossija (Mallarmé en Russie), Berne, Peter Lang Verlag, 1998.

5. À ce sujet, voir E. Etkind, « Baudelaire en langue russe », Europe, avril-mai 1967 et Adrian Wanner, Baudelaire in Russia, Gainesville, University Press of Florida, 1996. 
symbolistes de la seconde génération, l'affirmation de l'existence d'un monde transcendantal. Ivanov rédige un commentaire aux «Correspondances» dans l'article « Deux éléments du symbolisme contemporain » ${ }^{6}$ et le poème joue un rôle important dans sa théorie du symbolisme réaliste. Quant à Blok, il est familier du poète français, même s'il ne le traduit pas ${ }^{7}$ et nombreux sont les accents baudelairiens dans son œuvre, en particulier dans tout ce qui tourne autour de la représentation de la ville. Georges Nivat souligne à juste titre la proximité de «L'Inconnue » (« Neznakomka ») et du sonnet des Fleurs du mal, « À une passante $»^{8}$.

Mais, parmi les poètes français, c'est peut-être à Verlaine que revient la place centrale dans ce travail d'appropriation poétique. Certes, il est une figure emblématique surtout pour la première génération des symbolistes russes. On se souvient que, dans sa déclaration programme de 1892, « Des causes de la décadence et des nouveaux courants de la littérature russe contemporaine », Merežkovskij le prend comme antipode de Zola, comme symbole de l'anti-naturalisme et comme étendard des nouveaux courants. Merežkovskij met en scène un Verlaine stéréotypé « incarnant la révolte de la poésie contre l'hégémonie de la prose, de la forme brève contre le gros roman, du spirituel contre le social, de la 'pauvreté' contre l'Académie, de la 'musique' contre l'idée. » ${ }^{9}$ Les trois grands noms attachés au Verlaine russe sont ensuite des poètes de la première génération : Brjusov, Sologub et Annenskij ${ }^{10}$. Mais l'importance de Verlaine se fait sentir bien au-delà de la revendication explicite dont il est l'objet de la part des premiers symbolistes et semble plus prégnante que celle de Baudelaire parce qu'elle inspirerait en profondeur l'écriture symboliste même. Le très célèbre " Art poétique » de Verlaine ${ }^{11}$ est le ferment de la nouvelle esthétique et peut faire figure de texte-programme pour tout le symbolisme, en particulier dans ces deux exhortations : «De la musique avant toute chose » et « Pas la Couleur, rien que la nuance ! »

Il ne faudrait pas, en effet, réduire le symbolisme russe à l'expression de thèmes nouveaux (culte de l'instant, du moi, problématiques existentielles, fuite hors du quotidien, goût pour l'exotisme, plus tard existence de deux univers...) : le mouvement a aussi été recherche d'un nouveau langage poétique pour exprimer la radi-

6. «Dve stihii v sovremennom simvolizme », in Po zvezdam (En suivant les étoiles), SaintPétersbourg, 1909, p. 247-290.

7. Sa mère et sa grand-mère, en revanche, l'ont traduit, voir A. Wanner, op. cit.

8. E. Etkind et al., ed., Histoire de la littérature russe. 3 : Le XX $X^{\mathrm{e}}$ siècle. $1: L^{\prime} \hat{A} g$ e d'argent, Paris, Fayard, 1987, p. 145.

9. Hélène Henry, «Verlaine, poète russe. 'Un étranger si familier' », Slavica occitana, 10, 2000, p. 180.

10. Ibid. Dans cette remarquable étude, l'auteur compare les différents «Verlaine » russes. Brjusov donne la préférence au Verlaine de Romances sans paroles, privilégie le Verlaine technicien, auteur de pièces brèves, artisan impeccable de formes nouvelles. Il en fait un lyrique (proche de Fet) et un poète impressionniste. Sologub et Annenskij s'intéressent plus à Verlaine en ce qu'il correspond à leurs propres aspirations, poésie plus inquiète chez Sologub et qui rejoindrait la liberté pouchkinienne. Le Verlaine d'Annenskij est violemment décadent, noir, étouffant, tragique.

11. 1874, publié en 1884 dans Jadis et naguère. 
cale nouveauté de l'homme moderne, en proie à des sensations inconnues jusqu'alors. Pour les symbolistes, il n'existe pas de mots pour rendre compte de cette expérience inédite : voilà pourquoi la poésie des mots précis, employés dans leur sens lexical, est appelée à céder la place à une poésie des allusions, de l'inexprimable. Aussi l'émergence du symbolisme russe est-elle indissociable d'une remise en cause de l'usage traditionnel de la langue. À expérience inédite, écriture nouvelle. La poésie du mot exact, lexical, doit céder la place à une poésie des allusions, du non-dit, de l'inexprimable, de l'ineffable, d'où des effets suggestifs particuliers sur le lecteur, d'où la création d'un climat, d'une atmosphère ${ }^{12}$. Si elle n'était pas nouvelle pour la littérature européenne, cette revendication des potentialités suggestives du langage était neuve en terrain russe. Cette écriture suggestive s'appuie sur une sorte de brouillage sémantique qui fait émerger des sens secondaires, plus flous, occasionnels, produits par le contexte, d'où la mobilisation de tout un ensemble de procédés qui vont contribuer à cet estompage des contours, comme le remplissage sémantique arbitraire de structures syntaxiques stables, chez Blok $^{13}$. Pour Mihail Gasparov, l'ensemble de ces procédés culmine en un nouveau trope, canonisé par les symbolistes russes et qui vise à produire un élargissement du sens, c'est l'anti-emphase. Quand, dans «L'Inconnue», Blok écrit «Un soleil m'est confié », il est clair qu'il ne s'agit pas (ou pas seulement) de l'astre diurne. Lorsque, à un autre endroit, il déclare, sans préambule : «Seuls sur le ciel noir crissaient les fils du télégraphe », on peut simplement dire que ces « fils » suggèrent beaucoup de choses à la fois (la tristesse, le caractère mystérieux, le sentiment d'infini, l'hostilité...), mais toujours de façon approximative ${ }^{14}$. Simple procédé poétique ou «fenêtre sur l'éternité », le symbole est le mot tout désigné pour exprimer ce nouveau trope. Ce brouillage sémantique conduit aussi à privilégier les sons, quitte à les remplir d'un nouveau contenu' ${ }^{15}$, d'où l'importance de tous les procédés d'euphonie, de répétitions sonores, d'où le rôle privilégié de la musique. On voit ainsi que les exigences de Verlaine, «musique avant toute chose » et «nuance », sont en accord avec les recherches expressives des symbolistes et leur

12. Voir les déclarations de Brjusov dans les recueils Russkie simvolisty (Les symbolistes russes) ; M. L. Gasparov, « Poetika 'serebrjanogo veka' » (La poétique de 1' Ấge d'argent'), in Russkaja poezija serebrjanogo veka 1890-1917 (La poésie russe de l'Âge d'argent 1890-1917), Moscou, Nauka, 1993, p. 15.

13. Iouri Tynianov, Le vers lui-même : problème de la langue du vers, tr. fr., Paris, 10/18, 1977, p. 118-119 : " 'Dans les tavernes, les ruelles, les méandres /En un rêve électrique éveillé, / J'ai recherché sans fin les êtres beaux / Et immortellement épris de gloire'. Dans le cadre d'une construction rythmico-syntaxique habituelle de la strophe sont insérés des mots qui ont l'air fortuit ; il en résulte une proposition que l'on dirait sémantiquement ouverte ; le cadre 'Dans les tavernes,... j'ai recherché' est rempli par des membres secondaires qui ne peuvent être réunis par leur signification principale. Toute la force résulte de la stabilité du schéma rythmico-syntaxique et de l'instabilité sémantique de ce qui le remplit." On retrouve ce même procédé dans «L'Inconnue » où la préposition «au-dessus » relie une suite de substantifs (« restaurants » « poussière » « ennui » « lac ») hétérogènes.

14. M. L. Gasparov, art. cit.,p. 16.

15. Voir les célèbres allitérations de Bal’mont, du type «Čuždyj čaram černyj čeln... » (ce qui signifie mot à mot «Une barque noire insensible aux charmes...») ou encore ses fameux « Kamyši » (Les roseaux). 
volonté de substituer aux liens logiques des associations reposant sur l'émotion grâce à la musique. Andrej Belyj écrit : «La musique exprime de façon idéale le symbole. Le symbole pour cette raison est toujours musical. »16

Ce travail sur la langue est indissociable d'une recherche sur la forme: la période symboliste correspond à un renouvellement spectaculaire du mètre russe, à un enrichissement de la culture du vers ${ }^{17}$. La période est proche, de ce point de vue, de l'époque romantique russe et même, par certains aspects, du XVIII siècle et du travail des grands législateurs, Lomonosov et Trediakovskij qui ont posé les fondements de la poésie russe moderne. Cet enrichissement s'exprime de plusieurs façons : d'abord par une réactivation des formes métriques russes classiques. Le tétramètre iambique est largement renouvelé par Belyj et Brjusov. Des vers iambiques et trochaïques de longueur inhabituelle (plus de six pieds) sont utilisés. Dans le cadre d'une seule œuvre, longue ou brève («Snežnaja maska » - « Masque de neige » de Blok, 1907), on voit alterner différents mètres. Les poètes recourent aussi à des formes classiques du patrimoine mondial, de préférence gréco-latin et roman, mais aussi oriental. Le sonnet connaît une fortune exceptionnelle, ce qui s'explique par la place privilégiée que tient la poésie française dans l'essor poétique du tournant du siècle ${ }^{18}$. Cultivé par Baudelaire, Mallarmé, Verlaine, le sonnet est surtout le genre par excellence du Parnasse. Dès sa sortie en 1893, les « Trophées » de José Maria de Heredia ont fait l'objet de nombreuses traductions. Les plus grands noms de l'époque, Bal'mont, Ivanov, Brjusov, Vološin..., pratiquent abondamment le sonnet. Ces formes étrangères sont utilisées aussi bien dans le domaine de la traduction que pour l'écriture de textes russes originaux : elles ont alors valeur de citation, participant à la réappropriation d'un patrimoine culturel commun .

Mais la poésie symboliste a aussi expérimenté. En terrain russe, cela signifie la généralisation du vers tonique (dol'nik, puis taktovik) dont on trouve déjà des exemples chez les symbolistes (recueil «Kormčie zvezdy»- «Les astres pilotes » de Ivanov), même si cette tendance s'est surtout développée chez leurs successeurs. Proche du dol'nik, est aussi l'emploi de logaèdes (nombre de syllabes non accentuées variable mais identique d'un vers à l'autre, combinaison de pieds binaires et ternaires), surtout en traduction (la strophe saphique ${ }^{19}$ ). La rime, la strophe sont aussi l'objet de recherches particulières et d'un renouvellement significatif.

Globalement, la poésie des symbolistes est une poésie difficile, volontairement pensée pour une élite et nettement occidentalisée (titre des recueils en grec ou latin, vers écrits directement en allemand ou en grec chez Ivanov, etc.). Par rapport à l'histoire du vers russe, la période inaugurée par les symbolistes se caractérise par

16. A. Belyj, «Simvolizm kak miroponimanie» (Le symbolisme comme conception du monde) cité par M. L. Gasparov, art. cit., p. 29.

17. M. L. Gasparov, art. cit., p. 14-15.

18. Cela tient également au rôle de P. Buturlin.

19. M. L. Gasparov, Očerk istorii russkogo stiha (Essai d'histoire du vers russe), Moscou, 1984, p. 206-224. 
le recul de la métrique syllabo-tonique au profit du vers accentuel, il s'agit d'une transformation profonde, comparable à celle qui s'est déroulée au XVIII siècle (1730-1740) et a vu le vers syllabo-tonique s'imposer face au vers syllabique : le vers russe ne sonne plus comme avant.

De la pratique de nouveaux mètres à des essais d'analyse et de théorie, il n'y a qu'un pas que les principaux symbolistes franchirent sans hésiter, se faisant commentateurs de leurs propres expériences ou des vers russes classiques. Brjusov, Ivanov, Belyj composèrent ainsi des ouvrages de métrique: Cours abrégé de science du vers (Kratkij kurs nauki o stihe), Fondements de métrique (Osnovy stihovedenija), Essais sur la métrique, le rythme, l'euphonie, l'harmonie, la strophe et les formes (Opyty po metrike i ritmike, po evfonii i sozvučijam, po strofike $i$ formam) ${ }^{20}$ de Brjusov, et, pour Belyj, Le Symbolisme (Simvolizm), «La prose littéraire » («O hudožestvennoj proze »), plus tard, Le rythme comme dialectique (Ritm kak dialektika) ${ }^{21}$ pour ne citer que quelques titres. Ivanov faisait de véritables cours de métrique, n'hésitait pas, craie à la main, à expliquer la composition de tel ou tel poème ${ }^{22}$. En général, on ne pense pas spontanément à cet aspect essentiel de l'héritage symboliste, tant l'image dominante attachée au mouvement est celle de sa base philosophique et culturelle (Schopenhauer, Nietzsche, Wagner, Solov'ev...)

Plus largement, en s'intéressant ainsi aux formes poétiques, aux questions d'écriture, les symbolistes ont contribué à réhabiliter en Russie les préoccupations esthétiques générales et curieusement ont jeté les bases d'une approche radicalement neuve du texte, nourrissant tout le renouveau de la critique littéraire russe du XXe siècle, tel qu'il s'est exprimé principalement dans l'école formelle (19151930). Certes, tout un pan important de la critique littéraire symboliste était une critique de type essayiste, philosophique ou historico-culturel (Ajhenval'd, Merežkovskij, Ivanov à l'occasion). Et les formalistes ne manqueront pas de polémiquer plus tard contre elle, mais les symbolistes ont aussi été ceux qui ont suscité l'émergence d'une approche descriptive, analytique et scientifique de la littérature, en particulier grâce aux travaux d'Andrej Belyj sur le vers russe.

En quoi a consisté le travail de Belyj ? Je me limiterai ici à évoquer son rôle fondateur dans la réflexion sur le rythme poétique et l'utilisation de méthodes mathématiques, mais il va de soi que son apport au renouveau de la critique et de la théorie littéraires ne se limite pas à cela : tout aussi importants furent son intérêt pour Potebnja, ses réflexions générales sur le langage, l'image, etc. Le point de

20. Parus à Moscou respectivement en 1919, 1924, 1918.

21. Respectivement : 1910 ; Gorn (Le creuset), 1-2, 1919, p. 49-55 ; 1929.

22. L'Académie poétique ou Société des zélateurs du verbe (Poetičeskaja akademija ili ob̌̌čstvo revnitelej hudožestvennogo slova) fut inaugurée en 1909, lors de séances qui se déroulaient à la Tour et, plus tard, dans les locaux de la revue Apollon. Ces séances réunissaient les grands noms de la poésie de l'époque (Ivanov, Annenskij, Brjusov, Kuz'min, Blok) et un public de « jeunes » dont la plupart des acméistes. À ce sujet, voir, entre autres, M. L. Gasparov «Lekcii V. Ivanova o stihe v Poetičeskoj Akademii 1909 g. » (Cours de V. Ivanov sur le vers à l'Académie poétique en 1909), Novoe Literaturnoe Obozrenie (abrégé infra en NLO), 10, 1994, p. 89-105. 
départ de sa démarche est l'attention portée au rythme, et non au pied, comme le faisait la métrique traditionnelle. Ce changement de perspective tient au développement du vers tonique qui reste vers, alors qu'il n'y a pas de schéma métrique préétabli. Dans un premier temps, Belyj a défini le rythme en poésie comme l'ensemble des écarts par rapport à la norme. Il a cherché ensuite à donner une description et une analyse objectives de ce rythme poétique, fondées sur des données quantitatives, à partir d'un corpus important de tétramètres iambiques, empruntés à une vingtaine de poètes russes. Il a aussi voulu donner une représentation visuelle de ces écarts ${ }^{23}$, ses fameuses « figures » (toit, toit renversé, carré, escalier, losange, croix...), obtenues en reliant tous les pyrrhiques (pieds non accentués). Selon Belyj, plus il y a de figures et plus le rythme, donc le poème, est riche. Pour lui, plus l'écart par rapport à la norme est important et plus l'oeuvre a de valeur, ce qui constitue une rupture par rapport aux appréciations antérieures qui privilégiaient au contraire le respect de la norme. Ensuite, de la comparaison de ces figures, il a cherché à tirer des conclusions sur l'usage du iambe, chez un poète donné, entre différents poètes et finalement est arrivé à des conclusions générales sur l'évolution du iambe entre le XVIII' et le XIX ${ }^{e}$ siècle. Au XVIII siècle, l'accent est plutôt omis sur le deuxième pied, au XIX ${ }^{\mathrm{e}}$ siècle - plutôt sur le premier pied $^{24}$. On voit l'intérêt heuristique de cette démarche : il s'agit d'appuyer sur des observations matérielles, quantitatives, des remarques, généralement faites de façon empirique et impressionniste (richesse, variété, musique...). Cette façon de procéder n'était pas une innovation complète : elle est héritée de la philologie classique qui se sert d'études de ce genre pour dater les textes anciens. L'étude systématique des mètres utilisés dans telle ou telle œuvre ancienne (plus ou moins d'accents, place des accents), permettait sa datation, offrait la possibilité de classer par ordre chronologique les textes d'un auteur et d'étudier l'évolution de son style. Belyj, qui a eu au début du siècle un prédécesseur en la personne de Lev Polivanov, reprend cette méthode qu'il va généraliser en s'appuyant sur un décompte systématique, et surtout en utilisant les outils statistiques. (N'oublions pas qu'il est le fils d'un éminent professeur de mathématiques de l'Université de Moscou et qu'il a reçu luimême une formation scientifique). Mihail Gasparov écrit :

Pour la première fois et avec une telle ampleur des observations métriques étaient étayées par des calculs statistiques : d'un art, l'étude du vers devenait une science, comme la physique après les calculs de Galilée et la chimie après ceux de Lavoisier ${ }^{25}$.

23. À ce stade, Belyj avait une conception statique du rythme (figures), qu'il aura tendance à remplacer par une conception dynamique, le rythme étant défini par contraste, en mesurant l'écart entre deux vers de même rythme (résultats transcrits par des courbes).

24. Ce qui sera confirmé par les travaux ultérieurs.

25. M. L. Gasparov « Belyj-stihoved i Belyj-stihotvorec » (Belyj-théoricien et Belyj-poète), in A. Belyj, Problemy tvorčestva (Les problèmes de la création), Moscou, 1988, p. 445 ; Id., Sovremennyj russkij stih. Metrika i ritmika (Le vers russe contemporain. Métrique et rythme), Moscou, Nauka, 1974, p. 5. 
Ces premières études de Belyj, consacrées au tétramètre iambique russe, ont été publiées dans l'énorme volume Le Symbolisme, en $1910^{26}$. Ce livre, composé de nombreux autres articles d'une orientation toute différente (symbolisme théurgique de Belyj), a cependant joué un rôle décisif sur ceux qui allaient se faire connaître comme «formalistes », précisément à cause de ces articles de métrique. À propos de cet ouvrage, B. Eichenbaum écrit à ses parents (1910) :

Le contenu principal du livre, c'est l'analyse du rythme poétique. Il y a beaucoup de choses neuves, intéressantes et importantes au plus haut point. Beaucoup de points communs avec mes propres réflexions et mes propres intérêts. C'est peut-être le premier livre véritable, consacré, en russe, à la théorie du mot et je suis sûr qu'il va marquer le début de toute une époque. Tous les procédés de la critique antérieure, historique, publicistique, psychologique, impressionniste, doivent être mis à l'écart ou se défaire de leur affreux dilettantisme et se mêler aux autres sciences plus générales. [...] En un mot, c'est un livre remarquable²7.

Jusqu'à sa mort, Belyj a poursuivi ses recherches sur le vers russe. En 1910-1911, il animait, aux éditions Musagète, un cercle d'études métriques sur le pentamètre iambique qui devaient déboucher sur un « Manuel de rythme » (Učebnik ritma), resté, hélas ! à l'état de manuscrit mais qui constituait un pas en avant considérable par rapport aux articles parus dans Le Symbolisme ${ }^{28}$. Après son retour de l'étranger en 1916, Belyj commença à élaborer sa théorie du « geste rythmique » qui devait être à la base de ses derniers travaux et en particulier de son étude, encore trop peu assimilée par la critique, Le rythme comme dialectique ${ }^{29}$ (1929). Il n'entre pas dans mon propos d'analyser dans le détail l'ensemble de ses travaux sur le vers, ni de me prononcer sur leur validité scientifique ${ }^{30}$ : je voudrais simplement insister sur leur rôle de ferment pour le développement de la théorie de la littérature dans la Russie

26. Articles ayant trait à ce domaine: «Lirika i eksperiment» («Poésie lyrique et expérimentation »), «Opyt harakteristiki russkogo 4-stopnogo jamba » (Essai de caractérisation du tétramètre iambique russe), "Sravnitel'naja morfologija ritma russkih lirikov » (Morphologie comparative du rythme des poètes lyriques russes), « Ne poj, krasavica, pri mne... » (Ne chante pas en ma présence, beauté...), p. 231- 428 et 567-633. Sur l'écriture de ces articles, voir A. Belyj, Meždu dvuh revoljucij (Entre deux révolutions), Leningrad, Izd. pisatelej v Leningrade, 1934, p. 352-353 et 376-378.

27. Eichenbaum, «Pis'ma roditeljam» (Lettres à ses parents), Olga Eichenbaum, ed., Revue des Études slaves, 57 (1), 1985, p. 24-25.

28. Ces éléments ont été révélés en premier dans la publication capitale de S. S. Grečiškin et A. V. Lavrov «O stihovedčeskom nasledii A. Belogo » (L'héritage métrique de Belyj), qui contient plusieurs inédits dont un fragment de ce «manuel », Trudy po znakovym sistemam (Travaux sur les systèmes de signes), 12, Tartu, 1981, p. 97-146.

29. C'est sans doute en raison des conclusions extrêmes sur la signification révolutionnaire du « Cavalier de bronze » auxquelles arrivait Belyj que cet ouvrage a eu tendance à être laissé dans l'ombre ; pourtant la conception dynamique du rythme qui sous-tend cette étude (voir supra) mérite d'être relevée, voir M. L. Gasparov, «Belyj-stihoved... », art. cit., p. 445-446. On comparera cependant avec la publication récente d'une discussion de cet ouvrage par les philologues du GAHN, « Stih i smysl 'Mednogo vsadnika' » (Vers et signification du 'Cavalier de bronze'), M. V.Akimova, S. E. Ljapina, eds., Philologica, 5 (11-13), 1998, p. 255-276, qui conteste toute validité aux décomptes de Belyj.

30. Voir les travaux déjà cités de Grečiškin, Lavrov et de Gasparov. 
du $\mathrm{XX}^{\mathrm{e}}$ siècle, et en particulier sur leur importance pour les représentants de l'école formelle.

Belyj, par exemple, a fait le 14 octobre 1917 un exposé sur «le geste rythmique », au cercle Puškin de l'Université de Saint-Pétersbourg devant plusieurs formalistes, qui ont été à nouveau séduits par ses conceptions, déjà différentes de celles de $1910^{31}$. Au début des années 20, les travaux de Belyj (et de Brjusov) sont aussi discutés au cercle linguistique de Moscou en présence de Tomaševskij ${ }^{32}$, Brik, Jakobson... La somme de Tomaševskij sur le vers russe qui paraît en 1929, Du vers (O stihe), s'inscrit dans la tradition des travaux de Belyj, même si c'est pour en contester la validité : le livre discute aussi les conceptions de Brjusov. Mais il y a encore plus intéressant : Tomaševskij avait visiblement élaboré une méthode proche de celle de Belyj, bien qu'avec des différences, indépendamment du poète et au même moment, ainsi qu'il s'en ouvre à Brjusov (lettres des années 1908-191233). Il était donné aux deux hommes de se rencontrer en 1933, quelques mois avant la mort de Belyjj ${ }^{34}$. Selon Holševnikov, Tomaševskij aurait même déclaré : «Il y a eu beaucoup de spécialistes du vers de talent, mais un seul de génie, - Belyj. »35

Dans son article rétrospectif sur la méthode formelle (1927), Tomaševskij écrira même :

C'est à l'école symboliste que nous sommes redevables de la réhabilitation des problèmes de l'art pur dans l'étude de la littérature. Les essais critiques et historiques d'A. Biély, de V. Ivanov, de V. Brioussov ont attiré notre attention sur l'élément que les traditionalistes laissaient dans l'ombre.

Et la note 1 précise :

Dans ses études du recueil Le symbolisme, 1910, A. Biély a sous certains rapports devancé les formalistes [c'est moi qui souligne. CD]. Ses recherches sur le vers iambique russe ont beaucoup influencé les travaux postérieurs. Sa notion d'esthétique expérimentale contenait un germe du formalisme ${ }^{36}$.

31. Ce n'est bien sûr pas le seul exposé de Belyj, il en fit plus de trente entre 1917 et 1920, S. S. Grečiškin, A. V. Lavrov, art. cit., p.108-109.

32. Séance du 1er avril 1921, «Tomaševskij i Moskovskij lingvističeskij kružok » (Tomaševskij et le Cercle linguistique de Moscou), publication de L. Fleishman, Trudy po znakovym sistemam, 9, Tartu, 1977, p. 113-124. Tomaševskij, Brik et Jakobson ont aussi rédigé des comptes rendus des travaux de métrique de Belyj et de Brjusov.

33. «Pis’ma B. Tomaševskogo V. Brjusovu » (Lettres de Tomaševskij à Brjusov) ; publication de L. Fleishman, Trudy po znakovym sistemam , 5, Tartu, 1971, p. 532-544.

34. Voir « B. V.Tomaševskij v perepiske s A. Belym » (Correspondance de Tomaševskij avec Belyj), A. V. Lavrov, ed., in Puškinskij Dom (Maison Pouchkine), Leningrad, 1982, p. 224239.

35. M. L. Gasparov, « Belyj-stihoved... », art. cit., p. 445.

36. B. Tomachevski, « La nouvelle école d'histoire littéraire », Revue des Études slaves, 8 (34), 1928, p. 233. On se souvient aussi de ses propos de 1925 : «D'où est issu le formalisme ? Des articles de Belyj, du séminaire de Vengerov... » 
L'importance de Belyj pour l'étude quantitative du vers russe, pour les recherches sur le rythme est certes exceptionnelle, mais il n'est pas le seul dont les travaux méritent un examen. La position d'Ivanov, son attitude vis-à-vis de la méthode formelle ${ }^{37}$ seraient aussi intéressantes à étudier, tout comme l'héritage de Brjusov, peut-être un peu négligé jusqu'à présent. Il est à noter enfin que, dans les années 20 , les symbolistes ont eu tendance à apporter quelques correctifs à leurs travaux de métrique : est-ce sous l'influence de la critique des formalistes ${ }^{38}$ ?

Plus généralement, Belyj et les symbolistes ont initié en Russie un rapprochement décisif et particulièrement fécond entre l'art et la science, entre la poésie et la philologie, ce qu'illustrent à merveille les cas de Brjusov, Ivanov, Belyj, Annenskij... Mais, il est tout aussi important de rappeler que cette symbiose a fonctionné en sens inverse et que nombreux ont alors été les philologues, les érudits de profession, les universitaires comme T. Zelinskij, M. Rostovceff, E. Aničkov à s'intéresser aux problèmes de création. Au début du siècle, l'université de Saint-Pétersbourg est gagnée par l'effervescence artistique, générée par le symbolisme. Elle abrite de nombreux cercles étudiants de poésie; ses séminaires, celui de $\mathrm{S}$. Vengerov sur Puškin ou celui de K. D. Petrov, ressemblent beaucoup à des sociétés littéraires. Ce phénomène se concrétise par l'émergence de toute une génération de philologuespoètes, nés dans les années $1880-1890^{39}$, qui se partagera ensuite assez équitablement entre des poètes, N. Gumilev, O. Mandelstam... et des théoriciens de la littérature, V. Žirmunskij, B. Eichenbaum, V. Šklovskij, Ju. Tynjanov, pour ne citer que les plus célèbres. Trop souvent, ce rapprochement de la science et de l'art est présenté exclusivement comme l'apanage des « jeunes générations » du $\mathrm{XX}^{\mathrm{e}}$, des formalistes en particulier. Or, il fait partie sans doute d'un des aspects les plus féconds de l'héritage du symbolisme, dont les recherches sur le vers continuent à alimenter la vie philologique des années 20. Aussi conviendrait-il peut-être de revoir la chronologie en insistant sur la présence du ferment symboliste, même sur un mode mineur, dans la vie intellectuelle des années 20. Nous avons là un élément supplémentaire parlant en faveur d'une datation de l'Âge d'argent allant jusqu'au début des années $30^{40}$.

37. Objet d'un article de G. V. Obatnin et K. Ju. Postoutenko « V. Ivanov i formal'nyj metod » (V. Ivanov et la méthode formelle), Russkaja literatura, 1, 1992, p. 180-187. V. Ivanov a aussi fait un compte rendu de Poetika, 1919.

38. Brjusov apporte des correctifs dans sa deuxième édition de Osnovy stihovedenija (Fondements de métrique) ; les conceptions de Belyj connaissent également des infléchissements, enfin voir V. Ivanov « O novejših teoretičeskih iskanijah v oblasti hudožestvennogo slova » (À propos des derniers travaux dans le domaine du mot poétique), in Naučnye izvestija akademičeskogo centra Narkomprosa (Nouvelles scientifiques du centre académique du Narkompros), II, Moscou, 1922, p. 164-181.

39. Ce phénomène a été déjà étudié. Voir en guise de mise au point, notre article « L'université de Saint-Pétersbourg et 1'Âge d'argent », Saint-Pétersbourg : une fenêtre sur la Russie 19001935, E. Bérard, ed., Paris, Éditions de la Maison des Sciences de 1'Homme, 2000, p. 209-222 (version russe plus complète dans Sankt-Peterburg. Okno v Rossiju 1900-1935, Saint-Pétersbourg, Feniks 1997, p. 85-99). Parmi les travaux récents se rapportant à cette question, signalons la publication par A. V. Lavrov de la correspondance entre K. Močulskij et V. Žirmunskij, NLO, 35, 1999, p. 117-214.

40. 1930 : suicide de Majakovskij ; 1932-1933 : dernières lectures publiques de Mandelstam ; janvier 1934 : mort de Belyj. 
Bien plus, indépendamment de leur portée théorique générale, il importe de rappeler pour finir que les études métriques de Belyj sont à la base d'un des secteurs de pointe de la critique russe, l'étude quantitative du vers, appelée même à l'étranger «méthode russe ${ }^{41}$. Brillamment illustré par une pléiade de grands savants, tels que B. Tomaševskij, V.Žirmunskij, N. Troubetzkoy, R. Jakobson, K. Taranovsky ${ }^{42}$, A. Kolmogorov ${ }^{43}$..., ce domaine reste peut-être à l'heure actuelle un des secteurs les plus productifs de la critique littéraire savante en Russie, ainsi qu'en témoignent l'œuvre de Mihail Gasparov et la revue Philologica ${ }^{44}$.

Porté par tout un mouvement de renouveau philosophique, le symbolisme en Russie a aussi été réhabilitation de toutes les préoccupations d'esthétique et point de départ d'un formidable développement de la théorie littéraire : c'est sur cet aspect peut-être moins immédiatement présent aux consciences que j'ai souhaité insister ici.

\section{Université de Paris IV}

Centre Universitaire Malesherbes

108, Boulevard Malesherbes

75017 Paris

catherine.depretto@laposte.net

41. Voir M. L. Gasparov, « Kvantitativnye metody v russkom stihovedenii : itogi i perspektivy » (Les méthodes quantitatives dans les études métriques russes : bilan et perspectives), in id., Sovremennyj russkij stih, op. cit., p. 18-38.

42. Kiril Taranovsky (1911-1993) : a reçu sa formation de slaviste en Yougoslavie où il a soutenu en 1941 une thèse sur le rythme des mètres binaires russes, iambes et trochées (publiée en 1953) ; a enseigné à partir de la fin des années 50 aux États-Unis, à Berkeley puis à Harvard, a établi certaines lois concernant les mètres binaires russes et joué un rôle essentiel dans le développement des études sur Mandelstam, K. Taranovsky, Essays on Mandelstam, Cambridge, MA, Harvard University Press, 1976.

43. Sur A. Kolmogorov (1903-1987), éminent mathématicien, voir «Semiotičeskie poslanija » (Epîtres sémiotiques), NLO, 24, 1997, p. 21-246 et Vl. A. Uspenskij, « Predvarenie dlja čitatelej $N L O$ k semiotičeskim poslanijam Kolmogorova» (Avertissement aux épîtres sémiotiques de Kolmogorov, destiné aux lecteurs de NLO), ibid., p. 121-215.

44. Qui continue à paraître malgré d'énormes difficultés. 